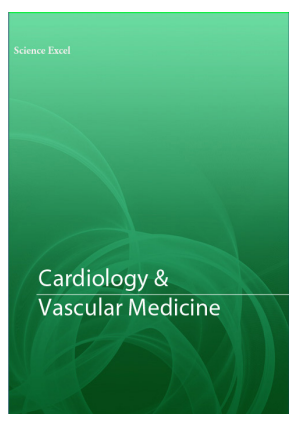

Correspondence

Ikram Krikez

Resident, Cardiology Department, University Hospital Center Lbn Rochd of Casablanca, Morocco

E-mail: Krikezikram@gmail.com

\footnotetext{
- Received Date: 28 April 2020;

- Accepted Date: 09 May 2020;

- Publication Date: 15 May 2020.
}

Keywords

Breast cancer, Trastuzumab, Anthracyclines, Cardio-toxicity, Clinical risk score, Cardiooncology

\section{Abbreviations}

CRS : Cardiotoxicity Risk Score; ASCO : American Society of Clinical Oncology; HER-2: Human Epidermal Growth Factor Receptor-2; LVEF: Left Ventricular Ejection Fraction; LV: Left Ventricule; LGS: the Longitudinal Global Strain; RT: Radiotherapy; CTRCD: cancer therapeutics-related cardiac dysfunction; CVD: Cardiovascular disease; HF: Heart failure; TIC: Trastuzumab-induced cardiotoxicity

Copyright

(c) 2020 Science Excel. This is an openaccess article distributed under the terms of the Creative Commons Attribution 4.0 International license.

\title{
Anthracyclines and Trastuzumab-induced Cardio- toxicity in Breast Cancer Patients: Testing a Clinical Risk Score in the First Cardio-Oncology Unit in Morocco
}

\author{
Krikez $I^{1 *}$, Benmalek $\mathbf{R}^{1}$, Maaroufi A', Bendahou $\mathrm{H}^{1}$, Abouriche A $^{1}$, Bennouna $\mathbf{G}^{1}$, \\ Drighil A', Azzouzi L' ${ }^{1}$, Habbal R' ${ }^{1}$, Haffadi $\mathbf{M}^{2}$, Tawfiq $\mathrm{N}^{2}$ and Benider A $^{2}$ \\ 'Cardio-Oncology Unit, Cardiology Deparment, University Hospital Center Lbn Rochd of Casablanca, Morocco \\ 2Oncology Department, Mohamed V Cancer Treatment Center, University Hospital Center Lbn Rochd, Casablanca, Morocco
}

\begin{abstract}
Background: Recent advances in the early detection and treatment of cancer have led to a significant improvement of cancer survival worldwide. However, long-term cardio-toxic side effects affect both patient survival and quality of life.

Aim: To assess the utility of the Cardio-toxicity Risk Score (CRS) to predict cardio-toxicity among our patients.

Material and Methods: We conducted a prospective observational study for 3 years in the first cardio-oncology unit in Morocco. For each breast cancer candidate to an adjuvant treatment with anthracyclines and/or trastuzumab, we calculated The CRS proposed by the ASCO, and classified the patients in 2 groups (high and low risk). We then performed sensitivity and correlation analysis between cardio-toxicity and the patients' CRS.

Results: In total, 413 patients were included, and when applying the CRS to them, 136 (32.9\%) were considered at high risk and 277 (67.1\%) at low risk. During follow-up, 42 patients (10.1\%) experienced cardio-toxicity. Sensitivity analysis showed that the CRS applied to our population had a sensitivity of $83 \%$ [ $95 \% \mathrm{Cl}: 0.78,0.92]$, a specificity of $65 \%$ [95\% Cl: $0.54,0.71]$, with a positive predictive value of $21 \%$ [95\% Cl: $0.08,0.39]$ and a negative predictive value of $97 \%$ [ $95 \% \mathrm{Cl}: 0.90,0.99]$. On the other hand, correlation analysis found a significant positive correlation with both transient and permanent cardio-toxicity.

Conclusion: The CRS demonstrated good sensitivity and negative predictive value for the development of cardio-toxicity in our population, suggesting that intensive cardiac monitoring may not have as much interest in low-risk patients that in high-risk patients.
\end{abstract}

\section{Introduction}

Breast cancer is becoming an increasingly urgent problem worldwide. In fact, it is the world's most common cancer among women according to the International Agency for Research on Cancer (IARC) latest global cancer data [1]. In 2018, 24.2\% and about one in 4 of all new cancer cases diagnosed in women worldwide are breast cancer. Moreover, it is the leading cause of cancer death in women $(15.0 \%)[1,2]$.

In Morocco, it is also by far the most common cancer in women with an incidence of $19.2 \%$ in 2018 (10 136 new cases), and the second cause of death by cancer after lung cancer, according to the same source [1].

Over these last decades, Cancer survival and patients' outcome significantly improved by major advances in treatment options especially in targeted agents such as Trastuzumab, a humanized monoclonal antibody against
HER2 [3], and also in early detection and screening of its short and long-term side-effects.

However, despite these advances, morbidity associated with breast cancer treatment affect both patient's survival and quality of life [4].

Cardio-toxicity is one of the most feared and undesirable side effect of cancer treatments, occurring in approximately $10 \%$ of the patients [5], It is usually multifactorial, due to combined protocols as adjuvant chemotherapy especially anthracycline- based regimen, trastuzumab and radiation therapy. Its onset depends also on other parameters like the patient's predisposition or risk (comorbidities, history, and medication's intake).

Currently, although several risk scores have been proposed [6,7], among which the clinical risk score (CRS), developed by the American Society of Clinical Oncology (ASCO) [8], and the Framingham risk score (FRS) [9], it is difficult to predict which patients are at highest 
risk of developing cardio-toxicity from chemotherapy or targeted therapy .

The aim of our study was to assess the utility of the CRS to predict anthracyclines and trastuzumab-induced cardio-toxicity in breast cancer patients in a Moroccan Cardio-oncology unit in order to implement a standardized risk score in routine clinical practice.

\section{Material and Methods}

This was a prospective observational study, conducted in the cardio-oncology unit of Casablanca, the first cardio-oncology unit in Morocco, from November 2017 to December 2019. We included all new cases of early-stage (I-III) breast cancer patients, who are candidates to receive an adjuvant treatment with anthracyclines associated or not with trastuzumab in the HER2+ patients.

We excluded from our study patients having metastatic disease, or a cardiac disease at the time of the diagnosis.

For all our patients, a baseline cardiac assessment was performed, and we collected information concerning their medical history (with Data on demographics, cancer characteristics, cardiac risk factors etc.), in addition to clinical examination, biological exams including Troponins I, Brain natriuretic peptides (BNP) and lipid level. We also performed an electrocardiogram and an echocardiography with assessment of Left Ventricular Ejection Fraction (LVEF) and Longitudinal Global Strain (LGS).

After this baseline work-up, we assessed our patients' risk accordingly to the ASCO CRS in order to define patients at increased risk for developing a cardiac dysfunction, considering the following criteria [8]:

- Treatment that includes any of the following:

- High-dose anthracycline (eg, doxorubicin $\geq 250 \mathrm{mg} / \mathrm{m}^{2}$, epirubicin $\geq 600 \mathrm{mg} / \mathrm{m}^{2}$ )

- High-dose Radiotherapy (RT) ( $\geq 30$ Gy) where the heart is in the treatment field.

- Low-dose anthracycline (eg, doxorubicin $<250 \mathrm{mg} / \mathrm{m}^{2}$, epirubicin $\left.<600 \mathrm{mg} / \mathrm{m}^{2}\right)$ in combination with low-dose RT ( $<$ $30 \mathrm{~Gy}$ ) where the heart is in the treatment field.

- Treatment with low-dose anthracycline (eg, doxorubicin < $250 \mathrm{mg} / \mathrm{m}^{2}$, epirubicin $<600 \mathrm{mg} / \mathrm{m}^{2}$ ) or trastuzumab alone, and presence of any of the following risk factors:

- Multiple cardiovascular risk factors ( $\geq$ two risk factors), including smoking, hypertension, diabetes, dyslipidemia, and obesity, during or after the therapy's completion.

- Old age ( $\geq 60$ years) at the cancer treatment's time.

- Compromised cardiac function (eg, borderline low LVEF [50\% to $55 \%$ ], history of myocardial infarction, $\geq$ moderate valvular heart disease) at any time before or during treatment

- Treatment with low dose anthracycline (eg, doxorubicin $<250$ $\mathrm{mg} / \mathrm{m}^{2}$, epirubicin $<600 \mathrm{mg} / \mathrm{m}^{2}$ ) followed by trastuzumab (sequential therapy)

Following this criteria, we classified our patients in two groups: High risk and low risk of developing cancer therapeutics-related cardiac dysfunction (CTRCD)

Patients receiving anthracyclines and/or trastuzumab were followed prospectively from the treatment initiation's time, and according to the European Society of Cardiology 2016 guidelines [10].
For low-risk patients (normal baseline echocardiogram, no clinical risk factors), an assessment of cardiac function was performed after a cumulative total doxorubicin (or equivalent) dose of $200 \mathrm{mg} / \mathrm{m}^{2}$ or epirubicin $400 \mathrm{mg} / \mathrm{m}^{2}$ or even earlier if clinical symptoms and/or an increase in cardiac enzymes was observed, and then at 3,6,9,12 months after the end of the treatment. For the anti-HER2 therapy, cardiac monitoring was performed every 4 cycles of the treatment, and then every 3 and 6 months after its completion.

More frequent monitoring was performed for patients with abnormal baseline echocardiography (reduced or low normal LVEF, structural heart disease) and those with higher clinical risk at baseline (prior anthracyclines, previous myocardial infarction, and treated heart failure).

The importance of this follow-up was the screening and the early detection of CTRCD that was defined as a decrease of the LVEF more than $10 \%$, and below the lower limit of $50 \%$, with or without symptoms $[10,11]$. CTRCD was considered transient if the patient's LVEF returned to the baseline value during the study period, and permanent if the LVEF was sustained either below $50 \%$ or more than $10 \%$ below the baseline LVEF's value .

\section{Statistical analysis}

First, we performed a sensitivity analysis using SPSS 21.0. A true positive was defined as a patient in the high-risk score group who experienced a CTRCD during the follow-up period, and a true negative was defined as a low-risk patient who experienced no cardio-toxicity during the study. Using those cut-points, sensitivity and specificity, as well as positive and negative prediction values were calculated.

In the second analysis, we evaluated the correlation between the patients' risk and the degree of recovery of the LVEF using the Pearson correlation coefficient. We aimed to assess whether or not a high risk accordingly to the CRS was correlated to a permanent cardiotoxicity and if a low-risk was correlated with a transcient CTRCD.

\section{Ethical approval}

The participation in the study was voluntary; consent was free and clear, written or oral. The study was conducted in compliance with the ethical standards of the responsible institution on human subjects as well as with the Helsinki Declaration.

\section{Results}

During the study period, 413 new cases of early-stage breast cancer patients, who were candidates to receive an adjuvant treatment with anthracyclines, and/or trastuzumab, were referred to the cardio-oncology unit of Casablanca.

Their baseline characteristics are assessed in Table 1.

Mean age in the cohort was 51 years (range: 23-88 years; standard deviation: 11 years).

Concerning the cardiovascular risk factors, $10.1 \%$ had hypertension, $7 \%$ diabetes Mellitus, $15.5 \%$ dyslipidemia and more than half the women were menopausal. Almost $25 \%$ of our patients had non-cardiovascular associated comorbidities including asthma, dysthyroidism etc.

$41 \%$ of our patients had stage II disease at the time of diagnosis (169 (40.9\%)), and 94\% ( $\mathrm{n}=387)$ were candidates to adjuvant chemotherapy including Anthracyclines in $86 \%$ of cases. Median 


\begin{tabular}{|l|l|}
\hline \multicolumn{1}{|c|}{ Characteristics } & \multicolumn{1}{|c|}{ Value } \\
\hline Number of patients & 413 \\
\hline Mean age (years) & $51 \pm 11.7$ \\
\hline Mean Body Mass Index & $27.1 \pm 6$ \\
\hline Pre-existing cardiovascular conditions & \\
and risk factors [n (\%)] & \\
Hypertension & $42(10 ; 1 \%)$ \\
Diabetes mellitus & $29(7,0 \%)$ \\
Smoking & $5(1,2 \%)$ \\
Hyperlipidemia & $64(15,5 \%)$ \\
Menopause & $217(52,5 \%)$ \\
Coronary artery disease & $9(2,1 \%)$ \\
Significant valvular disease & $5(1,2 \%)$ \\
Renal failure & $11(2,6 \%$ \\
Stroke & $4(0,9 \%)$ \\
\hline Number of non-cardiovascular & \\
comorbidities [n (\%)] & \\
None & $305(73,9 \%)$ \\
1 to 2 & $103(24,9 \%)$ \\
>3 & $5(1,2 \%)$ \\
\hline Tumor stage [n (\%)] & \\
I & $71(17,2 \%)$ \\
II & $169(40,9 \%)$ \\
III & $82(19,9 \%)$ \\
Unknown & $91(22,0 \%)$ \\
\hline Chemotherapy [n (\%)] & $38 \% \pm 9 \%$ \\
Adjuvant & $387(93,7 \%)$ \\
Neoadjuvant & $26(6,3 \%)$ \\
Antracyclines based & $95(23,0 \%)$ \\
Trastuzumab & $34(8,2 \%)$ \\
Anthracyclines plus trastuzumab & $261(63,2 \%)$ \\
\hline Anthracyclines cumulative dose [n (\%)] & $31(7,5 \%)$ \\
High dose & $319(77,2 \%)$ \\
Low dose & $63(15,3 \%)$ \\
Unknown & $176(42,6 \%)$ \\
\hline Radiotherapy [n (\%)] & \\
\hline Radiotherapy cumulative dose n (\%)] & \\
High dose $>30$ Gy & \\
Low dose $<30$ Gy & $(10,7 \%)$ \\
Unknown & \\
\hline Mean baseline LVEF & \\
\hline Mean baseline LGS & \\
\hline
\end{tabular}

Table 1. Patients' characteristics at baseline

\begin{tabular}{|l|l|l|}
\hline Variables & $\begin{array}{l}\text { Clinical risk } \\
\text { score }\end{array}$ & \\
\hline & Low-risk & High-risk \\
\hline Patients [n (\%)] & $277(67,1 \%)$ & $136(32,9 \%)$ \\
\hline CTRCD [n (\%)] & $7(16,6 \%)$ & $35(83,4 \%)$ \\
\hline Transient CTRCD [n (\%)] & $7(100 \%)$ & $24(68,5 \%)$ \\
\hline $\begin{array}{l}\text { Permanent CTRCD [n } \\
(\%)]\end{array}$ & $0(0 \%)$ & $11(31,5 \%)$ \\
\hline
\end{tabular}

Table 2. Cancer therapeutics-related cardiac events stratified according to the CRS

\begin{tabular}{|l|l|l|}
\hline \multicolumn{1}{|c|}{ Variables } & \multicolumn{1}{c|}{ Value } & \multicolumn{1}{c|}{ CI 95\% } \\
\hline Sensitivity & 0.83 & {$[0.78,0.92]$} \\
\hline Specificity & 0.65 & {$[0.54,0.71]$} \\
\hline Positive predictive value & 0.21 & {$[0.08,0.39]$} \\
\hline Negative predictive value & 0.97 & {$[0.90,0.99]$} \\
\hline
\end{tabular}

$\mathrm{CI}=$ confidence interval

Table 3. Sensitivity analysis.

\begin{tabular}{|l|l|l|l|l|}
\hline & \multicolumn{2}{|c|}{ Low-risk } & \multicolumn{2}{c|}{ High- risk } \\
\hline & $\begin{array}{l}\text { Pearson } \\
\text { correlation }\end{array}$ & $\mathrm{p}$ value & $\begin{array}{l}\text { Pearson } \\
\text { correlation }\end{array}$ & $\mathrm{p}$ value \\
\hline $\begin{array}{l}\text { Transient } \\
\text { CTRCD }\end{array}$ & 0.092 & 0.062 & 0.354 & $<0.001$ \\
\hline $\begin{array}{l}\text { Permanent } \\
\text { CTRCD }\end{array}$ & -- & -- & 0.236 & $<0.001$ \\
\hline
\end{tabular}

Table 4. Correlation analysis between CRS and CTRCD reversibility.

baseline LVEF was 58\% (standard deviation 9\%), and mean baseline LGS in absolute value was $21 \%$ (standard deviation $4 \%$ ).

When applying the CRS to our study population, 136 of them (32.9\%) were considered at high risk and 277 (67.1\%) at low risk.

In the study period, 42 patients (10.1\%) experienced CTRCD among which $79,1 \%$ were asymptomatic and $20.9 \%$ had heart failure. Moreover, 31 patients $(73,8 \%)$ had full recovery of their LVEF.

The majority of patients who experienced CTRCD were in the high-risk group $(83.4 \%, \mathrm{n}=35)$ against 7 patients $(16.6 \%)$ in the low-risk group.

Also, $100 \%$ of the low-risk patients completely recovered their cardiac function during the 3 years follow-up versus $68.5 \%$ ( $n=$ 24 ) in the high-risk group, with permanent CTRCD in more than a third of our patients.

Table 2 details all the cases of cardio-toxicity in our patients stratified by the CRS and the degree of LVEF within 3 years of therapy initiation.

After this, we performed a sensitivity analysis where we calculated sensitivity and specificity, and also positive and negative prediction values (Table 3 ). The results showed that when applying the CRS to our population, the sensitivity was $83 \%$ [95\% CI: $0.78,0.92$ ], the specificity $65 \%$ [95\% CI: $0.54,0.71]$, with a positive predictive value of $21 \%$ [ $95 \%$ CI: $0.08,0.39$ ] and a negative predictive value of 97\% [95\% CI: 0.90, 0.99]

In the second analysis, we evaluated the correlation between the patients' risk and the degree of recovery of the LVEF (Table 4)

In high risk patients, the results showed a significant positive correlation with both transient and permanent CTRCD, with however, a significant correlation between high-risk patients and transient CTRCD (0.354, $\mathrm{p}<0.001)$. On the other hand, no significant correlation was assessed between low-risk patients and transient CTRCD (0.092, $\mathrm{p}=0.062)$. 


\section{Discussion}

Breast cancer is the most common cancer diagnosed in Morocco, with an estimated 10136 new cases in 2018 based on the Moroccan cancer registry, the second most common cancer in terms of mortality rate, responsible of $10.7 \%$ of deaths among cancer patients $[1,2]$. In cancer survivors, cardiovascular disease (CVD) is the second cause of long-term morbi-mortality [12] and the first cause of death among female survivors from breast cancer [13].

These past decades, advances in chemotherapy and targeted therapies significantly increased cancer patients' survival, hence the long-term side-effects of cancer therapy, especially chemotherapy. Some molecules like anthracyclines and herceptine were found to be associated with an important risk of cardiac damage, including left ventricular (LV) dysfunction and heart failure (HF), thromboembolic complications, hypertension as well as arrhythmias. Many breast cancer patients die of causes independent of the cancer itself but related to treatments cardiovascular side-effect, which draws attention to the importance of managing cardiovascular risk factors for the longterm care of patients diagnosed with breast cancer [13].

\section{Anthracyclines}

Anthracycline-based chemotherapy regimens used as adjuvant or neo-adjuvant in breast cancer treatment displayed survival improvement [14], however, they carry a well-known cytotoxicity on cardiomyocytes that may be responsible for a cardiomyopathy, that can often be irreversible [15].

The mechanism of anthracycline-induced cardiac injury has been the subject of several studies and yet has not been fully explained $[16,17]$.

The risk of cardio-toxicity secondary to anthracyclines is mostly dose-dependent and depends on the cumulative dose. For instance, doxorubicin is responsible of an incidence of congestive $\mathrm{HF}$ ranging from $3 \%$ to $5 \%$ with a cumulative dose of $400 \mathrm{mg} / \mathrm{m}^{2}$, from $7 \%$ to $26 \%$ at $550 \mathrm{mg} / \mathrm{m}^{2}$, and from $18 \%$ to $48 \%$ at $700 \mathrm{mg}$ / $\mathrm{m}^{2}[18,19]$.

Pein et al [20] analyzed 229 patients treated with anthracycline and reported a relative risk of HF of 1.93 in patients who received $250-400 \mathrm{mg} / \mathrm{m}^{2}$ compared to those treated with lower doses of anthracyclines, confirming a strong relationship between cumulative dose and cardio-toxicity.

\section{Trastuzumab}

Trastuzumab, is a monoclonal antibody directed against the HER2 that significantly improve survival in women with HER2positive early-stage breast cancer [21]. However, trastuzumabinduced cardiotoxicity (TIC), mainly reversible left ventricular dysfunction, may limit its use [22]. TIC severity is quite variable and can go from asymptomatic decline in LVEF to symptomatic $\mathrm{HF}$, and does not seem to be associated to either dose or duration [22]. It is usually a type 2 cardio-toxicity unlike anthracyclines that are classified among type 1 cardio-toxicity [23]. Indeed, contrary to anthracyclines that directly cause structural damage to cardiomyocytes, its mechanisms of action include cytotoxicity through inhibition of signal transduction, neoangiogenesis and repair of DNA damage caused by other treatments (type 2 cardiotoxicity) [23].

In Mantarro et al meta-analysis [24], including approximately 29000 patients, severe TIC was found in about $3 \%$ of patients, with an increasing incidence up to $19 \%$ among older patients, smokers and patients with diabetes, hypertension or cardiovascular disease. However, after TIC diagnosis, trastuzumab is often interrupted, which is associated with a higher rate of cancer recurrence $[25,26]$. In our study we found the same factors with similar proportions.

\section{Risk assessment}

Predicting patients at high risk of developing cardio-toxicity following a sequential anthracycline-based chemotherapy and trastuzumab still remains a challenge for the cardio-oncologist.

Although no guidelines are available, the strategy mostly used to reduce and to prevent cardio-toxicity is a precise analysis of cardiovascular risk factors or underlying subclinical cardiovascular damage in addition to an accurate assessment of the optimal therapy and maximal cumulative dose [27]. This was the approach we adopted in our unit, which allowed us to establish a database and results close to the literature series.

So far, few risk prediction scores have been published in the literature [27-29].

The CRS proposed by the ASCO takes both patient characteristics and treatment risk factors into consideration, however it doesn't include treatment doses and hasn't been tested in real life clinical prospective settings, therefore, it has not been validated nor recommended by savant societies [10].

In order to produce a clinical risk stratification model, the score should include treatment with dosage related risk factors; patient related risk factors and should be tested in real world clinical setting.

\section{Treatment related risk factors}

The various regimens used in treatment of breast cancer carries different risk of cardio-toxicity alongside with the dosage used [19].

Molecules used for chemotherapy are divided into 4 categories based on incidence of myocardial contractile damage; and group 4 alone accounts over $10 \%$ of cardio-toxicity. The most common breast cancer treatment used which belong to group 4 are Anthracyclines and Trastuzumab.

LV dysfunction seems to be more frequent in case of:

- High-dose anthracycline (doxorubicin $>250 \mathrm{mg} / \mathrm{m}^{2}$ or epirubicin $>600 \mathrm{mg} / \mathrm{m}^{2}$ ); observed in $7.5 \%$ in our series.

- Lower-dose anthracycline in combination with lowerdose radiation therapy ( $\mathrm{RT}<30 \mathrm{~Gy}$ ) where the heart is in the treatment field;

- Patients with cardiovascular risk factor including advanced age and structural heart abnormality;

- Sequential therapy: treatment with anthracycline followed by trastuzumab.

One of the limits of our study was the high proportion of the indeterminate status of Radiotherapy and anthracyclines cumulative doses (in $39.8 \%$ and $15.3 \%$ of the cases respectively).

\section{Patient-related risk factors}

Among patients' related risk factor, the ones most frequently reported in the literature are age of exposure $(<15$ or $>65$ year old), heart centered radiation therapy, obesity, hypertension and diabetes mellitus besides demographic features (female sex, black ethnicity) [29]. 
Combining more than 3 of these risk factors is related to 5 times higher risk of cardio-toxic adverse effects versus patients without any risk factors [29].

These risk factors are found in our series however it was difficult for us to calculate the relative risk of cardio-toxicity with the lack of appropriate statistical methodology.

\section{Detection and Monitoring of cardiotoxicity}

A clinical risk stratification score may represent an effective, inexpensive tool to help managing development of cardio-toxicity after treatment initiation and reduce early discontinuation of therapy by early detection and treatment.

Besides clinical risk factors, baseline and post chemotherapy strain imaging using echocardiography and cardiac biomarkers has been included in cardio-toxicity prediction. In this perspective, a group of experts from the American Society of Echocardiography and the European Association of Cardiovascular Imaging [30] defined cardio-toxicity as a decrease in LVEF (measured by the Simpson biplane method) of more than $10 \%$ from the reference value to a value below the lower limit of normal [31], as it was the case in our study. GLS is also a reproducible echocardiographic method to measure changes in left ventricular contractility before ejection fraction declines [30].

Cardiac biomarkers are a useful tool to identify patients with higher risk of toxicity. Elevated troponin level has been useful for detection of in the early cardio-toxicity related to chemotherapy $[32,33]$.

Based on clinical features, imaging and cardiac biomarkers, elaborating a prediction model that can enhance sensitivity to detect high risk patients to develop chemotherapy-related toxicity and should benefit from close intense monitoring.

On the other hand, it allows identifying low risk patients who will not need long term cardiac monitoring, saving its cost for high risk group.

Cardio-oncology clinicians and researchers should focus on assessment and applicability of such risk stratification model in more extensive prospective and long term settings. According to our study, the hypothesis that a high risk was positively correlated with permanent cardio-toxicity and that low risk had a negative correlation with transient CTRCD was not true in our analysis as shown in Table 4.

\section{Conclusion}

In conclusion, in our study, the CRS was proven to be an effective tool to assess breast cancer patients' risk and to predict the development of CTRCD. It demonstrated good sensitivity and negative predictive value for the development of cardio-toxicity in a real-world population of breast cancer patients undergoing anthracyclines and/or trastuzumab based therapy. These findings suggest that intensive cardiac monitoring may not have interest in low-risk patients, but that high-risk patients must benefit from early consultation in a cardio-oncology unit, due to the increased risk of cardiac events.

\section{Conflicts of interest}

The authors declare they have no conflict of interest.

\section{Authors' contributions}

All the authors contributed equally in the drafting of the manuscript. All the authors read and agreed to the final manuscript.

\section{Acknowledgements}

The authors would like to acknowledge Professor Rachida Habbal the head of the cardiology department who created the first cardio-oncology unit in Morocco and enabled this work.

We also like to thank Professor Nezha Tawfik and the oncology department team for their collaboration and the fact they entrusted their patients to our unit.

\section{References}

1. Bray F, Ferlay J, Soerjomataram I, Siegel RL, Torre LA, Jemal A. Global cancer statistics 2018: GLOBOCAN estimates of incidence and mortality worldwide for 36 cancers in 185 countries [published correction appears in CA Cancer J Clin. 2020 Jul;70(4):313]. CA Cancer J Clin. 2018;68(6):394-424

2. Coughlin SS, Ekwueme DU. Breast cancer as a global health concern. Cancer Epidemiol. 2009;33(5):315-318.

3. Viani GA, Afonso SL, Stefano EJ, De Fendi LI, Soares FV. Adjuvant trastuzumab in the treatment of her-2-positive early breast cancer: a meta-analysis of published randomized trials. BMC Cancer. 2007;7:153.

4. Binkley JM, Harris SR, Levangie PK, et al. Patient perspectives on breast cancer treatment side effects and the prospective surveillance model for physical rehabilitation for women with breast cancer. Cancer. 2012;118(8 Suppl):2207-2216.

5. Cardinale D, Colombo A, Bacchiani G, et al. Early detection of anthracycline cardiotoxicity and improvement with heart failure therapy. Circulation. 2015;131(22):1981-1988.

6. Romond EH, Jeong JH, Rastogi P, et al. Seven-year follow-up assessment of cardiac function in NSABP B-31, a randomized trial comparing doxorubicin and cyclophosphamide followed by paclitaxel (ACP) with ACP plus trastuzumab as adjuvant therapy for patients with node-positive, human epidermal growth factor receptor 2-positive breast cancer. J Clin Oncol. 2012;30(31):3792-3799.

7. Ezaz G, Long JB, Gross CP, Chen J. Risk prediction model for heart failure and cardiomyopathy after adjuvant trastuzumab therapy for breast cancer. J Am Heart Assoc. 2014;3(1):e000472.

8. Armenian SH, Lacchetti C, Barac A, et al. Prevention and Monitoring of Cardiac Dysfunction in Survivors of Adult Cancers: American Society of Clinical Oncology Clinical Practice Guideline. J Clin Oncol. 2017;35(8):893-911.

9. Law W, Johnson C, Rushton M, Dent S. The Framingham risk score underestimates the risk of cardiovascular events in the HER2-positive breast cancer population. Curr Oncol. 2017;24(5):e348-e353.

10. Zamorano JL, Lancellotti P, Rodriguez Muñoz D, et al. 2016 ESC Position Paper on cancer treatments and cardiovascular toxicity developed under the auspices of the ESC Committee for Practice Guidelines: The Task Force for cancer treatments and cardiovascular toxicity of the European Society of Cardiology (ESC) [published correction appears in Eur Heart J. 2016 Dec 24;:]. Eur Heart J. 2016;37(36):2768-2801.

11. Gavila J, Seguí MÁ, Calvo L, et al. Evaluation and management of chemotherapy-induced cardiotoxicity in breast cancer: a Delphi study. Clin Transl Oncol. 2017;19(1):91-104.

12. Mertens AC, Liu Q, Neglia JP, et al. Cause-specific late mortality among 5-year survivors of childhood cancer: the Childhood Cancer Survivor Study. J Natl Cancer Inst. 2008;100(19):13681379.

13. Patnaik JL, Byers T, DiGuiseppi C, Dabelea D, Denberg TD. Cardiovascular disease competes with breast cancer as the leading cause of death for older females diagnosed with breast cancer: a retrospective cohort study. Breast Cancer Res. 2011;13(3):R64.

14. Polychemotherapy for early breast cancer: an overview of the 
randomised trials. Early Breast Cancer Trialists' Collaborative Group. Lancet. 1998;352(9132):930-942.

15. Ades F, Zardavas D, Pinto AC, Criscitiello C, Aftimos P, de Azambuja E. Cardiotoxicity of systemic agents used in breast cancer. Breast. 2014;23(4):317-328.

16. Volkova $\mathrm{M}$, Russell $\mathrm{R}$ 3rd. Anthracycline cardiotoxicity: prevalence, pathogenesis and treatment. Curr Cardiol Rev. 2011;7(4):214-220.

17. Zhang $\mathrm{S}$, Liu $\mathrm{X}$, Bawa-Khalfe $\mathrm{T}$, et al. Identification of the molecular basis of doxorubicin-induced cardiotoxicity. Nat Med. 2012;18(11):1639-1642.

18. Swain SM, Whaley FS, Ewer MS. Congestive heart failure in patients treated with doxorubicin: a retrospective analysis of three trials. Cancer. 2003;97(11):2869-2879.

19. Von Hoff DD, Layard MW, Basa P, et al. Risk factors for doxorubicin-induced congestive heart failure. Ann Intern Med. 1979;91(5):710-717.

20. Pein F, Sakiroglu O, Dahan M, et al. Cardiac abnormalities 15 years and more after adriamycin therapy in 229 childhood survivors of a solid tumour at the Institut Gustave Roussy. Br J Cancer. 2004;91(1):37-44

21. Moja L, Tagliabue L, Balduzzi S, et al. Trastuzumab containing regimens for early breast cancer. Cochrane Database Syst Rev. 2012;2012(4):CD006243

22. Tripathy D, Slamon DJ, Cobleigh M, et al. Safety of treatment of metastatic breast cancer with trastuzumab beyond disease progression. J Clin Oncol. 2004;22(6):1063-1070.

23. Pondé NF, Lambertini $M$, de Azambuja E. Twenty years of anti-HER2 therapy-associated cardiotoxicity. ESMO Open. 2016;1(4):e000073.

24. Mantarro S, Rossi M, Bonifazi $M$, et al. Risk of severe cardiotoxicity following treatment with trastuzumab: a metaanalysis of randomized and cohort studies of 29,000 women with breast cancer. Intern Emerg Med. 2016;11(1):123-140.
25. Yu AF, Yadav NU, Lung BY, et al. Trastuzumab interruption and treatment-induced cardiotoxicity in early HER2-positive breast cancer. Breast Cancer Res Treat. 2015;149(2):489-495.

26. Suter TM, Procter M, van Veldhuisen DJ, et al. Trastuzumabassociated cardiac adverse effects in the herceptin adjuvant trial. J Clin Oncol. 2007;25(25):3859-3865.

27. Herrmann J, Lerman A, Sandhu NP, Villarraga HR, Mulvagh SL, Kohli M. Evaluation and management of patients with heart disease and cancer: cardio-oncology. Mayo Clin Proc. 2014;89(9):1287-1306.

28. Dranitsaris G, Rayson D, Vincent M, et al. The development of a predictive model to estimate cardiotoxic risk for patients with metastatic breast cancer receiving anthracyclines. Breast Cancer Res Treat. 2008;107(3):443-450.

29. Lotrionte $\mathrm{M}$, Biondi-Zoccai G, Abbate A, et al. Review and metaanalysis of incidence and clinical predictors of anthracycline cardiotoxicity. Am J Cardiol. 2013;112(12):1980-1984.

30. Plana JC, Galderisi M, Barac A, et al. Expert consensus for multimodality imaging evaluation of adult patients during and after cancer therapy: a report from the American Society of Echocardiography and the European Association of Cardiovascular Imaging. J Am Soc Echocardiogr. 2014;27(9):911939.

31. Lang RM, Badano LP, Mor-Avi V, et al. Recommendations for cardiac chamber quantification by echocardiography in adults: an update from the American Society of Echocardiography and the European Association of Cardiovascular Imaging. J Am Soc Echocardiogr. 2015;28(1):1-39.e14.

32. Cardinale D, Sandri MT, Colombo A, et al. Prognostic value of troponin I in cardiac risk stratification of cancer patients undergoing high-dose chemotherapy. Circulation. 2004;109(22):2749-2754

33. Cardinale D, Sandri MT, Martinoni A, et al. Left ventricular dysfunction predicted by early troponin I release after highdose chemotherapy. J Am Coll Cardiol. 2000;36(2):517-522. 